\title{
Wheel Wear Prediction of High-Speed Train Using NAR and BP Neural Networks
}

\author{
Ning Fan, Shuwen Wang*, Caixia Liu \\ College of Mechanical Engineering \\ University of Shanghai for Science and Technology \\ Shanghai, China \\ *corresponding author Email: wsw@usst.edu.cn
}

\author{
Xiaoming Liu \\ Institute of Mechanics \\ China Academy of Science \\ Beijing, China
}

\begin{abstract}
In this paper, the field measured wheel wear data of high-speed trains are studied by variance analysis, and prediction models are developed using NAR and BP neural networks. The results show that the wheel position has a significant effect on the wheel wear, and the position of the carriage has little influence on the wheel wear. The NAR neural network can be used to predict the dynamic change of wheel diameter and therefore to predict the wheel wear of high-speed trains. The wheel diameter data are classified and the range of wheel wear can be predicted by means of training the BP neural network.
\end{abstract}

Keywords-Big data; Wheel wear; Variance analysis; NAR neural network; Prediction

\section{INTRODUCTION}

With the increasing speed of passenger trains in China, the wheel wear and fatigue damage is becoming a more and more serious problem. Wheel wear not only increases the maintenance workload of the train and the railway operation cost, but also has a great influence on the driving stability and safety, comfort of passengers and service life of each part of the high-speed trains [1]. Therefore, scholars [2-6] have done a lot of research on wheel wear using three methods: numerical analysis, wheel-rail wear laboratory test and field measurement.

Compared with the numerical analysis and wear laboratory test methods, the field measured data present the actual situation of wheel wear. With the rapid development of modern detection technology and integrated automation technology of modern railway industry, the railway related departments of China have collected a large number of online and offline measurement data of wheel system, these data have rich information of wheel rolling system. Using these data information, predictive operation models of high-speed trains may be developed. Lin [7] studied the running surface wear of wheel-sets based on re-profiling measurement from 16 bogies of heavy haul locomotives at Malmbanan (Sweden). Lin [8] also carried out a reliability study using a Bayesian survival analysis framework to explore the impact of a locomotive wheel's installed position on its service lifetime and to predict its reliability characteristics. Pombo $[9,10]$ developed a computational tool that can be used to predict the evolution of the wheel profiles for a given railway system as a function of running distance.
The wheel wear of the CRH380BL train from Beijing to Shanghai is measured daily by Beijing Railway Bureau and a huge number of wheel wear data have been collected. In this paper, based on the measured wheel diameter from February 1 to March 1 in 2016, the wheel diameter variation trend and wear amount are predicted using NAR (Non-linear Auto Regressive) and BP (Back Propagation) neural networks in MATLAB, respectively. Neural networks are divided into static neural network and dynamic neural network. Static neural network such as BP neural network is widely used to simulate nonlinear mappings. About $80 \%$ neural networks used today are BP network. The main application of dynamic neural network is the prediction of time series. In this paper, the dynamic NAR neural network is used to predict the changing of wheel diameter. The conventional statistical wear model predicting wear as a function of mileage is not accurate because the actual operation condition is varied time from time. But, the models developed in this paper were based on the real measured wear data, which considering all operation conditions.

\section{VARIANCE ANALYSIS OF WHEEL WEAR LOSS}

\section{A. Effect of positions of Carriage and Wheel on Wheel Wear Loss}

CRH380BL type high speed train has 16 compartments, each carriage has two front and rear bogies, and each bogie has two sets of wheels. Every day the wheel diameter is measured by the Railway Bureau. The variance analysis was carried out on the reduction of wheel diameter to study the effect of carriage position and wheel position on the wear of each wheel.

The basic idea of variance analysis is to determine whether the influence of the factors on the research results is significant by comparing the deviation of the data and the random error at different levels. Suppose factor $A$ has $\mathrm{r}$ different levels $A_{1}, A_{2}, \cdots, A_{r}$; factor $B$ has s different levels $B_{1}, B_{2}, \cdots, B_{S}$. The result of the test under each $A$ and $B$ is $X_{i j}(i=1,2, \cdots, r ; j=1,2, \cdots, s)$. The error squared sum of $S_{e}$, effect squared sum $S_{A}$ and $S_{B}$ of $A$ and $B$ factors are calculated as: 


$$
\begin{aligned}
& S_{e}=\sum_{i=1}^{r} \sum_{j=1}^{s}\left(X_{i j}-\bar{X}_{i \cdot}-\bar{X}_{\cdot j}+\bar{X}\right)^{2} \\
& S_{A}=\sum_{i=1}^{r} \sum_{j=1}^{s}\left(\bar{X}_{i \cdot}-\bar{X}\right)^{2}=s \sum_{i=1}^{r}\left(\bar{X}_{i \cdot}-\bar{X}\right)^{2} \\
& S_{B}=\sum_{i=1}^{r} \sum_{j=1}^{s}\left(\bar{X}_{\cdot j}-\bar{X}\right)^{2}=r \sum_{j=1}^{s}\left(\bar{X}_{\cdot j}-\bar{X}\right)^{2}
\end{aligned}
$$

For the significance level $\alpha$, if

$$
F_{A}=\frac{S_{A} /(r-1)}{S_{e} /[(r-1)(s-1)]} \sim F((r-1),(r-1)(s-1)),
$$

factor $A$ has a significant effect on the objective. Similarly, if

$$
F_{B}=\frac{S_{B} /(s-1)}{S_{e} /[(r-1)(s-1)]} \sim F((s-1),(r-1)(s-1)),
$$

factor $B$ has a significant effect on the objective. $F_{A}$ and $F_{B}$ are the $F$ statistics for factors A and B.

Assuming that the carriage number is $A_{i}$ and the wheel position is $\mathrm{B}_{\mathrm{j}}$. By means of variance analysis it can be obtained that $\mathrm{F}_{\mathrm{A}}=1.445, \mathrm{~F}_{\mathrm{B}}=2.115, \mathrm{~F}_{1-0.1}(15,105)=1.56$, and $\mathrm{F}_{1-}$ ${ }_{0.1}(7,105)=1.78$. Because $F_{A}<F_{1-0.1}(15,105)$ and $F_{B}>F_{1-}$ $0.1(7,105)$, it is considered that the effect of factor $A$ on the wheel diameter is less than that of factor $B$, and the effect of factor B on wheel diameter is significant.

\section{B. The Effect of Bogie on Wheel Wear Loss}

The above method can be used to study the effect of bogie on the wheel wear loss. Assuming that the bogie is $\mathrm{C}_{1},(1=1,2)$, $\mathrm{C}_{1}$ represents the front bogie, and $\mathrm{C}_{2}$ stands for the rear bogie. Based on the data in Table 1, by means of variance analysis it can be obtained that $F_{C}=1.6, F_{1-0.1}(1,3)=5.54$. Because $F_{C}<F_{1-}$ $0.1(1,3)$, it may be concluded that the bogie position has no significant effect on the wheel wear loss.

TABLE 1 WHEEL WEAR LOSS OF FRONT AND REAR BOGIES

\begin{tabular}{|c|c|c|c|c|}
\hline \multirow{2}{*}{ Bogies } & \multicolumn{4}{|c|}{ Wheel No } \\
\cline { 2 - 5 } & 1 & 2 & 3 & 4 \\
\hline Front & 1.77 & 1.85 & 1.83 & 1.71 \\
\hline Rear & 1.60 & 1.80 & 1.85 & 1.69 \\
\hline
\end{tabular}

Considering the two variance analysis results, it may be concluded that the wheel position has a significant effect on the wear of wheel, but both the positions of carriage and bogie had no significant effect on the wear of wheel.

\section{PREDiction OF WHEEL DiAMETER CHANGING}

Because the wheel wear has nonlinear characteristics, NAR dynamic neural network can be used to model and simulate the wheel diameter changing. The NAR neural network model can be expressed by the following formula:

$$
y(\mathrm{t})=f(y(t-1), y(t-2), \cdots, y(t-n))
$$

It can be seen from Eq. (4) that $y(t)$ depends on its previous $\mathrm{n}$ data. The NAR neural network consists of input layer, hidden layer, output layer, input delay and output delay. Before using it, the output and output delay, and the number of hidden neurons are needed to be determined.
In this paper, the wheel diameter of wheel No. 1 in the carriage No. 1 is chosen as the object of study. Training data accounts for $70 \%$ of the total data, and both the verification data and test data account for $15 \%$. After several tests, the middle hidden node is 10 . Taking into account that there were multiple measurement errors in the measured data and their accuracy was limited to $0.1 \mathrm{~mm}$, the delay layers were set to 4 . For non-linear fitting, usually the S-type nonlinear transfer function is used as the activation function of the hidden layer, and the Tansig activation function is used as hidden layer of the section. However, since the value of the Tansig function is $[-1,1]$, only the existing data can be fitted and cannot be predicted. Therefore, in this work the Purelin linear function was used as the output layer to make the output value of the network be obtained arbitrarily. The structure of NAR neural network is shown in Fig. 1.

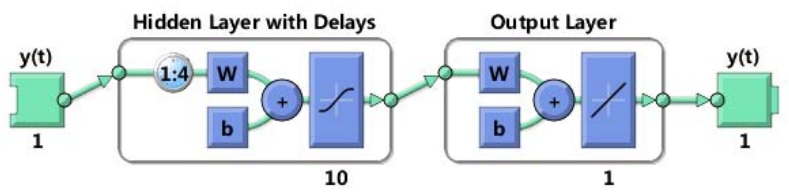

Fig. 1 The structure of NAR neural network

The results of the NAR dynamic neural network training are shown in Figs. 2 and 3. The vertical lines in Fig. 2 demonstrate the difference between the target value and the predicted value. A short line means that the training network has a good predictive accuracy. It can be seen from Fig. 3 that the error auto-correlation was the largest when the lag was 0 , and the error auto-correlation does not over the confidence interval when the lag was not 0 . Figures 2 and 3 demonstrate that the prediction of the wheel diameter changing using the NAR neural network model was reasonable good.

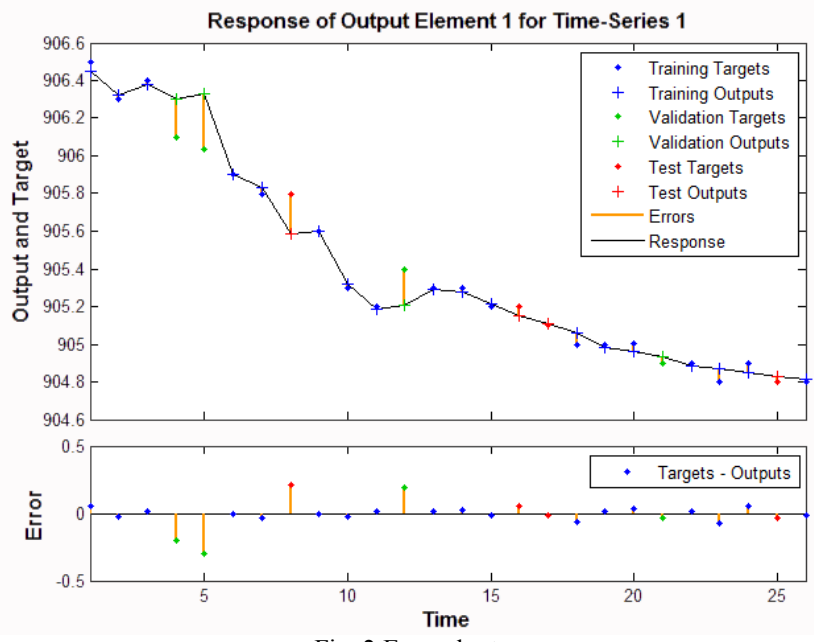

Fig. 2 Error chart 


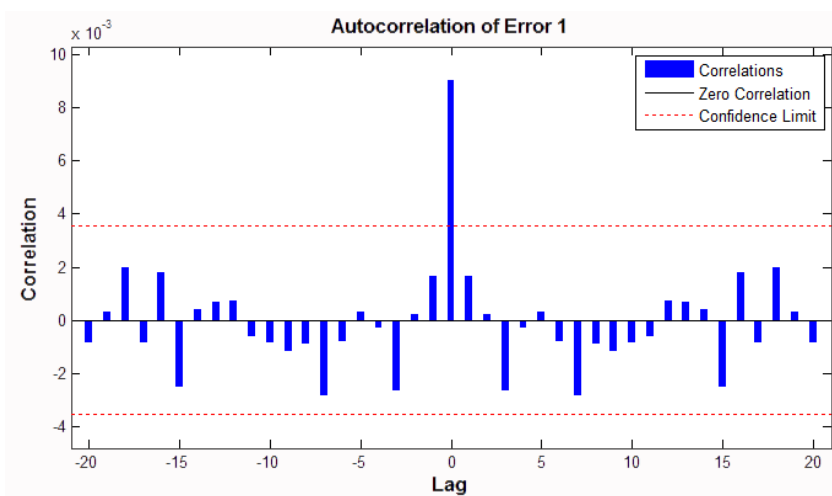

Fig. 3 Error auto-correlation

\section{WHEEL WEAR PATTERN IDENTIFICATION}

By means of the above variance analysis, it is known that both the positions of the wheel and the carriage have effect on wheel wear. At present, there are total 88 trains operation daily between Beijing and Shanghai and 36 CRH380BL high-speed trains. It is difficult to get the relationship between wheel wear and the positions of wheel and carriage by direct observation due to such a large number of measured data. But, this can be done by training BP network.

The main idea of the BP algorithm is to propagate the error of the output layer from the back forward (backward) layer by layer to indirectly calculate the hidden layer error. The algorithm is divided into two stages: in the first stage (forward propagation process) input information from the input layer through the hidden layer by layer to calculate the output value of each unit; in the second stage (reverse propagation process) output error layer by layer to calculate the hidden layer of the unit error, and use this error to correct the previous weights. The network weight adjustment is based on the Delta learning rule, that is, according to the gradient method along the error surface gradient of the most rapid decline, in order to achieve the minimum network error.

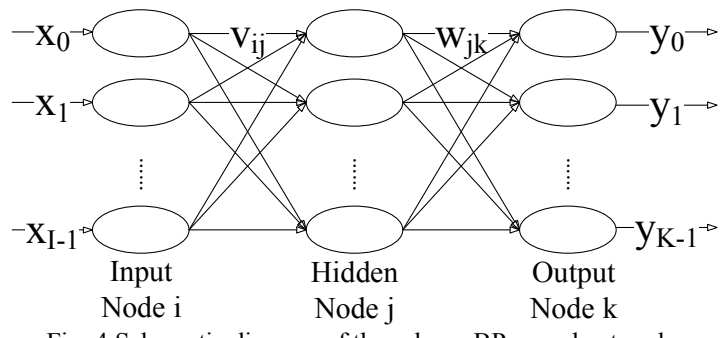

Fig. 4 Schematic diagram of three-layer BP neural network

Before the establishment of neural network, the data are needed to be processed. The input data are wheel position (18 ) and carriage number (1-16). Because the output of neural network pattern can only be identified as 0 and 1 , the values of measured wheel wear were set as 0 or 1 . The maximum wear of wheel is $2.5 \mathrm{~mm}$, the minimum wear of wheel is $1 \mathrm{~mm}$, and the majority wear is mainly concentrated in the range of 1.5 $\mathrm{mm}$ to $2 \mathrm{~mm}$. The wear loss is divided into 7 levels and each level is corresponding to a binary number as: $[1,1.5)$ is the first level, corresponding to $0000001 ;[1.5,1.6)$ is the second level, corresponding to $0000010 ;[1.6,1.7)$ is the third level, corresponding to $0000100 ;[1.7,1.8)$ is the fourth level, corresponding to 0001000 ; $[1.8,1.9)$ for the fifth level, corresponding to $0010000 ;[1.9,2.3)$ is the sixth level, corresponding to $0100000 ;[2.3,2.6)$ is the seventh level, corresponding to 1000000 .

According to empirical formula:

$s=\sqrt{0.43 m n+0.12 n^{2}+2.54 m+0.77 n+0.35}+0.51$

Where $\mathrm{S}$ is the number of hidden nodes, $\mathrm{m}$ is the number of input nodes, and $\mathrm{n}$ is the number of output nodes.

The network has 2 input nodes, 7 output nodes, and 5 hidden nodes. The network stimulus function is the Sigmiod function, and the initial value of the training learning rate was set as 0.1 , the momentum coefficient is 0.95 , and the maximum number of training is 10000 times. When the system error was less than 0.01 , it was considered that the network was convergent. The percentage of training samples, validated samples and test samples were $70 \%, 15 \%$, and $15 \%$, respectively.

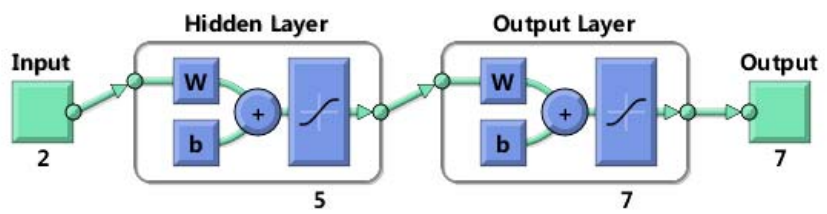

Fig. 5 Schematic diagram of the BP neural network

After 31 times training, the network converged and the mean square error was 0.09 . At this time, the recognition rate of training samples, verification samples, and test samples was $94.4 \%, 89.5 \%$, and $94.7 \%$, respectively, which basically meet the engineering needs. The training confusion matrix, verification confusion matrix, test confusion matrix, and total confusion matrix are illustrated in Tables 2 to 5 .

TABLE 2 TRAINING CONFUSION MATRIX

\begin{tabular}{|c|c|c|c|c|c|c|c|}
\hline 0 & 0 & 0 & 0 & 0 & 0 & 0 & NaN\% \\
$0 \%$ & $0 \%$ & $0 \%$ & $0 \%$ & $0 \%$ & $0 \%$ & $0 \%$ & NaN\% \\
\hline 0 & 0 & 0 & 0 & 0 & 0 & 0 & NaN\% \\
$0 \%$ & $0 \%$ & $0 \%$ & $0 \%$ & $0 \%$ & $0 \%$ & $0 \%$ & NaN\% \\
\hline 2 & 0 & 42 & 0 & 0 & 1 & 0 & $93.3 \%$ \\
$2.2 \%$ & $0 \%$ & $46.7 \%$ & $0 \%$ & $0 \%$ & $1.1 \%$ & $0 \%$ & $6.7 \%$ \\
\hline 0 & 0 & 2 & 43 & 0 & 0 & 0 & $95.6 \%$ \\
$0 \%$ & $0 \%$ & $2.2 \%$ & $47.8 \%$ & $0 \%$ & $0 \%$ & $0 \%$ & $4.4 \%$ \\
\hline 0 & 0 & 0 & 0 & 0 & 0 & 0 & NaN\% \\
$0 \%$ & $0 \%$ & $0 \%$ & $0 \%$ & $0 \%$ & $0 \%$ & $0 \%$ & NaN\% \\
\hline 0 & 0 & 0 & 0 & 0 & 0 & 0 & NaN\% \\
$0 \%$ & $0 \%$ & $0 \%$ & $0 \%$ & $0 \%$ & $0 \%$ & $0 \%$ & NaN\% \\
\hline 0 & 0 & 0 & 0 & 0 & 0 & 0 & NaN\% \\
$0 \%$ & $0 \%$ & $0 \%$ & $0 \%$ & $0 \%$ & $0 \%$ & $0 \%$ & NaN\% \\
\hline $0 \%$ & NaN\% & $95.5 \%$ & $100 \%$ & NaN\% & $0 \%$ & NaN\% & $94.4 \%$ \\
$100 \%$ & NaN\% & $4.5 \%$ & $0 \%$ & NaN\% & $100 \%$ & NaN\% & $5.6 \%$ \\
\hline
\end{tabular}


TABLE 3 VALIDATION CONFUSION MATRIX

\begin{tabular}{|c|c|c|c|c|c|c|c|}
\hline 0 & 0 & 0 & 0 & 0 & 0 & 0 & NaN\% \\
$0 \%$ & $0 \%$ & $0 \%$ & $0 \%$ & $0 \%$ & $0 \%$ & $0 \%$ & NaN\% \\
\hline 0 & 0 & 0 & 0 & 0 & 0 & 0 & NaN\% \\
$0 \%$ & $0 \%$ & $0 \%$ & $0 \%$ & $0 \%$ & $0 \%$ & $0 \%$ & NaN\% \\
\hline 0 & 1 & 10 & 0 & 0 & 0 & 0 & $90.9 \%$ \\
$0 \%$ & $5.3 \%$ & $52.6 \%$ & $0 \%$ & $0 \%$ & $0 \%$ & $0 \%$ & $9.1 \%$ \\
\hline 0 & 0 & 0 & 7 & 0 & 0 & 1 & $87.5 \%$ \\
$0 \%$ & $0 \%$ & $0 \%$ & $36.8 \%$ & $0 \%$ & $0 \%$ & $5.3 \%$ & $12.5 \%$ \\
\hline 0 & 0 & 0 & 0 & 0 & 0 & 0 & $\mathrm{NaN} \%$ \\
$0 \%$ & $0 \%$ & $0 \%$ & $0 \%$ & $0 \%$ & $0 \%$ & $0 \%$ & $\mathrm{NaN} \%$ \\
\hline 0 & 0 & 0 & 0 & 0 & 0 & 0 & $\mathrm{NaN} \%$ \\
$0 \%$ & $0 \%$ & $0 \%$ & $0 \%$ & $0 \%$ & $0 \%$ & $0 \%$ & $\mathrm{NaN} \%$ \\
\hline 0 & 0 & 0 & 0 & 0 & 0 & 0 & $\mathrm{NaN} \%$ \\
$0 \%$ & $0 \%$ & $0 \%$ & $0 \%$ & $0 \%$ & $0 \%$ & $0 \%$ & $\mathrm{NaN} \%$ \\
\hline $\mathrm{NaN} \%$ & $0 \%$ & $100 \%$ & $100 \%$ & $\mathrm{NaN} \%$ & $\mathrm{NaN} \%$ & $0 \%$ & $89.5 \%$ \\
$\mathrm{NaN} \%$ & $100 \%$ & $0 \%$ & $0 \%$ & $\mathrm{NaN} \%$ & $\mathrm{NaN} \%$ & $100 \%$ & $10.5 \%$ \\
\hline
\end{tabular}

TABLE 4 TEST CONFUSION MATRIX

\begin{tabular}{|c|c|c|c|c|c|c|c|}
\hline 0 & 0 & 0 & 0 & 0 & 0 & 0 & NaN\% \\
$0 \%$ & $0 \%$ & $0 \%$ & $0 \%$ & $0 \%$ & $0 \%$ & $0 \%$ & NaN\% \\
\hline 0 & 0 & 0 & 0 & 0 & 0 & 0 & NaN\% \\
$0 \%$ & $0 \%$ & $0 \%$ & $0 \%$ & $0 \%$ & $0 \%$ & $0 \%$ & NaN\% \\
\hline 0 & 0 & 8 & 0 & 0 & 0 & 0 & $100 \%$ \\
$0 \%$ & $0 \%$ & $42.1 \%$ & $0 \%$ & $0 \%$ & $0 \%$ & $0 \%$ & $0 \%$ \\
\hline 0 & 0 & 0 & 10 & 1 & 0 & 0 & $90.9 \%$ \\
$0 \%$ & $0 \%$ & $0 \%$ & $52.6 \%$ & $5.3 \%$ & $0 \%$ & $0 \%$ & $9.1 \%$ \\
\hline 0 & 0 & 0 & 0 & 0 & 0 & 0 & $\mathrm{NaN} \%$ \\
$0 \%$ & $0 \%$ & $0 \%$ & $0 \%$ & $0 \%$ & $0 \%$ & $0 \%$ & $\mathrm{NaN} \%$ \\
\hline 0 & 0 & 0 & 0 & 0 & 0 & 0 & $\mathrm{NaN} \%$ \\
$0 \%$ & $0 \%$ & $0 \%$ & $0 \%$ & $0 \%$ & $0 \%$ & $0 \%$ & $\mathrm{NaN} \%$ \\
\hline 0 & 0 & 0 & 0 & 0 & 0 & 0 & $\mathrm{NaN} \%$ \\
$0 \%$ & $0 \%$ & $0 \%$ & $0 \%$ & $0 \%$ & $0 \%$ & $0 \%$ & $\mathrm{NaN} \%$ \\
\hline $\mathrm{NaN} \%$ & $\mathrm{NaN} \%$ & $100 \%$ & $100 \%$ & $0 \%$ & $\mathrm{NaN} \%$ & $\mathrm{NaN} \%$ & $94.7 \%$ \\
$\mathrm{NaN} \%$ & $\mathrm{NaN} \%$ & $0 \%$ & $0 \%$ & $100 \%$ & $\mathrm{NaN} \%$ & $\mathrm{NaN} \%$ & $5.3 \%$ \\
\hline
\end{tabular}

TABLE 5 ALL CONFUSION MATRIX

\begin{tabular}{|c|c|c|c|c|c|c|c|}
\hline 0 & 0 & 0 & 0 & 0 & 0 & 0 & NaN\% \\
$0 \%$ & $0 \%$ & $0 \%$ & $0 \%$ & $0 \%$ & $0 \%$ & $0 \%$ & NaN\% \\
\hline 0 & 0 & 0 & 0 & 0 & 0 & 0 & NaN\% \\
$0 \%$ & $0 \%$ & $0 \%$ & $0 \%$ & $0 \%$ & $0 \%$ & $0 \%$ & NaN\% \\
\hline 2 & 1 & 60 & 0 & 0 & 1 & 0 & $93.8 \%$ \\
$1.6 \%$ & $0.8 \%$ & $46.9 \%$ & $0 \%$ & $0 \%$ & $0.8 \%$ & $0 \%$ & $6.3 \%$ \\
\hline 0 & 0 & 2 & 60 & 1 & 0 & 1 & $93.8 \%$ \\
$0 \%$ & $0 \%$ & $1.6 \%$ & $46.9 \%$ & $0.8 \%$ & $0 \%$ & $0.8 \%$ & $6.3 \%$ \\
\hline 0 & 0 & 0 & 0 & 0 & 0 & 0 & NaN\% \\
$0 \%$ & $0 \%$ & $0 \%$ & $0 \%$ & $0 \%$ & $0 \%$ & $0 \%$ & NaN\% \\
\hline 0 & 0 & 0 & 0 & 0 & 0 & 0 & NaN\% \\
$0 \%$ & $0 \%$ & $0 \%$ & $0 \%$ & $0 \%$ & $0 \%$ & $0 \%$ & NaN\% \\
\hline 0 & 0 & 0 & 0 & 0 & 0 & 0 & NaN\% \\
$0 \%$ & $0 \%$ & $0 \%$ & $0 \%$ & $0 \%$ & $0 \%$ & $0 \%$ & NaN\% \\
\hline $0 \%$ & $0 \%$ & $96.8 \%$ & $100 \%$ & $0 \%$ & $0 \%$ & $0 \%$ & $93.8 \%$ \\
$100 \%$ & $100 \%$ & $3.2 \%$ & $0 \%$ & $100 \%$ & $100 \%$ & $100 \%$ & $6.3 \%$ \\
\hline
\end{tabular}

The threshold values of the input layer to the hidden layer and the hidden layer are:

$$
\left[\begin{array}{cc}
2.1413 & 0.6719 \\
0.0047 & -8.298 \\
0.123 & -8.4408 \\
-0.1749 & 6.994 \\
-0.0066 & -6.4992
\end{array}\right] \text { and }\left[\begin{array}{c}
-3.9207 \\
-1.2902 \\
6.5262 \\
6.9563 \\
1.0681
\end{array}\right]
$$

The weights of hidden layer to the output layer and the output layer threshold are:

$$
\left[\begin{array}{ccccc}
2.7309 & 0.9073 & -2.2063 & -1.4267 & -1.7308 \\
1.2589 & 1.9912 & -1.3258 & -1.6424 & -2.1964 \\
1.2855 & 4.7701 & 2.0616 & 2.9132 & -5.1989 \\
-0.8498 & -3.0176 & -5.1678 & -3.5308 & 6.5662 \\
0.5206 & -4.5689 & 0.4916 & -1.2959 & 2.1583 \\
2.2635 & 1.846 & -0.843 & -1.1886 & -1.9047 \\
0.0431 & 3.4984 & -0.2745 & 6.3082 & 0.8743
\end{array}\right] \text { and }\left[\begin{array}{c}
-1.7071 \\
-1.5641 \\
-1.628 \\
2.5132 \\
-2.5381 \\
-0.2744 \\
0.0864
\end{array}\right]
$$

By means of the above trained network, the wheel wear of carriage No. 1 and wheel No. 1 may be predicted as shown in Table 6 and 7, respectively. The predicted wheel wear of wheel No. $1,4,5$, and 8 in carriage No. 1 are $[1.7,1.8)$, and the wheel No. of $2,3,6$, and 7 in carriage No. 1 are $[1.8,1.9)$. This indicates that the position of wheel had significant effect on the wheel wear.

TABLE 6. WHEEL WEAR PREDICTION RESULTS OF CARRIAGE NO.
\begin{tabular}{|c|c|c|}
\hline Wheel position & Prediction results code & Wear range \\
\hline $\mathbf{1}$ & 0001000 & {$[1.7,1.8)$} \\
\hline $\mathbf{2}$ & 0010000 & {$[1.8,1.9)$} \\
\hline $\mathbf{3}$ & 0010000 & {$[1.8,1.9)$} \\
\hline $\mathbf{4}$ & 0001000 & {$[1.7,1.8)$} \\
\hline $\mathbf{5}$ & 0001000 & {$[1.7,1.8)$} \\
\hline $\mathbf{6}$ & 0010000 & {$[1.8,1.9)$} \\
\hline $\mathbf{7}$ & 0010000 & {$[1.8,1.9)$} \\
\hline $\mathbf{8}$ & 0001000 & {$[1.7,1.8)$} \\
\hline
\end{tabular}

TABLE 7. WHEEL WEAR PREDICTION OF WHEEL NO. 1 IN ALL 16 CARRIAGES

\begin{tabular}{|c|c|c|}
\hline Carriage No. & Prediction results code & Wear range \\
\hline $\mathbf{1}$ & 0001000 & {$[1.7,1.8)$} \\
\hline $\mathbf{2}$ & 0001000 & {$[1.7,1.8)$} \\
\hline $\mathbf{3}$ & 0001000 & {$[1.7,1.8)$} \\
\hline $\mathbf{4}$ & 0001000 & {$[1.7,1.8)$} \\
\hline $\mathbf{5}$ & 0001000 & {$[1.7,1.8)$} \\
\hline $\mathbf{6}$ & 0001000 & {$[1.7,1.8)$} \\
\hline $\mathbf{7}$ & 0001000 & {$[1.7,1.8)$} \\
\hline $\mathbf{8}$ & 0001000 & {$[1.7,1.8)$} \\
\hline $\mathbf{9}$ & 0001000 & {$[1.7,1.8)$} \\
\hline $\mathbf{1 0}$ & 0001000 & {$[1.7,1.8)$} \\
\hline $\mathbf{1 1}$ & 0001000 & {$[1.7,1.8)$} \\
\hline $\mathbf{1 2}$ & 0001000 & {$[1.7,1.8)$} \\
\hline $\mathbf{1 3}$ & 0001000 & {$[1.7,1.8)$} \\
\hline $\mathbf{1 4}$ & 0001000 & {$[1.7,1.8)$} \\
\hline $\mathbf{1 5}$ & 0001000 & {$[1.7,1.8)$} \\
\hline $\mathbf{1 6}$ & 0001000 & {$[1.7,1.8)$} \\
\hline
\end{tabular}

The predicted wear results of wheel No. 1 in all 16 carriages are shown in Table 7 . The wear of each wheel No. 1 in all carriages is in the range of $[1.7,1.8)$, indicating that the position of carriage had no significant effect on the wear of wheel. 


\section{CONCLUSIONS}

In this paper, wheel wear prediction models are developed using NAR and BP neural networks, and the predicted wheel wear are verified by measured wheel diameters of CRH380BL high-speed trains traveling between Beijing and Shanghai. Rail operators can record data in the EXCEL table, and then use the network in MATLAB to predict wear. The following conclusions may be obtained:

(1) The wheel position had a significant effect on wheel wear, but the position of carriage had no significant effect on wheel wear.

(2) The NAR neural network can be used to predict the dynamic change of wheel diameters.

(3) By training the BP neural network, the wheel diameter data may be classified to obtain the wheel wear range at each position. There were only two wear conditions for eight wheels of each carriage in this study.

\section{ACKNOWLEDGMENT}

The authors gratefully acknowledge the financial support from the National Natural Science Foundation of China (No. 51275126).

\section{REFERENCES}

[1] X. Li, X. Jin, D. Hu, Calculation model of wheel wear and its numerical method [J]. Chinese Journal of Mechanical Engineering, 2009, 45(9): 193-200.

[2] X. Li, Z. Wen, X. Jin, Study on wheel wear and rolling contact fatigue of heavy haul railway [J]. Railway Transaction of China, 2011, 33(3): 28-34.

[3] J. Ding, F. Li, Y. Huang, Calculation of wheel wear based on half Hertz contact [J]. Journal of Southwest Jiao Tong University, 2011, 46(2):195199.

[4] H. Hu, K. Lu, W. Shao, Investigation and Research on the Datong Qinhuangdao railway wagon wheel wear problem [J]. Railway Transaction of China, 2010, 32(1):30-37.

[5] P. Shao, M. Fu, Y. Zhou, Study on wheel wear of heavy haul railway freight train based on Archard model[J]. Railway rolling stock, 2012, 32(2):42-45.

[6] L. Gou, Tracking research on wheel wear of high speed train [D]. Beijing Jiaotong University, 2011.

[7] Lin J, Asplund M, Nordmark T. Data analysis of wheel-sets' running surface wear based on re-profiling measurem. [C]// The, International Heavy Haul Association Conference. 2016:95-102.

[8] Lin J, Asplund M, Parida A. Reliability Analysis for Degradation of Locomotive Wheels using Parametric Bayesian Approach[J]. Quality \& Reliability Engineering, 2014, 30(5):657-667.

[9] Pombo J, Ambrósio J, Pereira M, et al. Development of a wear prediction tool for steel railway wheels using three alternative wear functions[J]. Wear, 2011, 271(1-2):238-245.

[10] Pombo J, Ambrósio J, Pereira M, et al. A railway wheel wear prediction tool based on a multibody software[J]. Journal of Theoretical \& Applied Mechanics, 2010, 48(3):751-770. 